\title{
On the Environmental and Economic Impact of Utility-scale Renewable Energy Deployment
}

\author{
Aaqib Peerzada \\ Texas A\&M University \\ peerzada@tamu.edu
}

\author{
Miroslav Begovic \\ Texas A\&M University \\ begovic@ece.tamu.edu
}

\author{
Dejan Ostojic \\ The World Bank \\ dostojic@worldbank.org
}

\begin{abstract}
As per the U.S Energy Information Administration's latest inventory of electricity generators, renewable energy, most notably solar and wind, will account for roughly $70 \%$ of nearly 40 gigawatts of new electricity generating capacity to start commercial operation in 2021. The year 2021 will also set a record in the deployment of utility-scale solar capacity by adding 15.4 gigawatts of capacity to the grid, which surpasses the 12 gigawatts increase in 2020. The rapid increase of renewable energy is expected to significantly decrease emissions of greenhouse gases and change the load profile in the power grid by suppressing production from conventional generators. This paper aims to propose a framework to study the impact of utility-scale solar $P V$ deployment on the generation resource allocation and investigate the economics and policy of electricity generation and carbon emissions. The investigation is carried on the generation resource pool of the southeast region of the U.S augmented by a substantial amount of utility-scale solar generation.
\end{abstract}

\section{Introduction}

Over the past decade the deployment of renewable energy especially solar and wind has dominated the addition of new generation capacity in the United States [1]. Even though the renewable energy appears to be the most popular new addition to the existing energy mix across many states in the U.S, the intermittent nature of such generation adds a new layer of complexity to the operation and planning of the power grid. One example of such a complexity is the concept of system net load. Traditionally the system load profile has been extensively used for power system management applications. However, the accelerating expansion of the renewables has rendered system load profile less informative and thus inadequate for most operation and planning applications. Instead, system net load, which is defined as the demand that must be met by dispatchable (non-intermittent) sources has gained popularity. An interesting recent work to estimate the system net load is presented in [2].
The system net load exhibits much faster changes than the traditional load profile when viewed in subhourly time scales. The changes are more pronounced when viewed at a very high temporal resolution. This is due to the fact that the output of the renewables like solar and wind is intermittent and thus imposing a variable generation pattern on the system. This has an important consequence on the resource allocation of the dispatchable sources; the output of which needs to be regulated at much shorter timescales to meet the rapid changes in the system net load. Responding to such rapid changes may even incentivize the utilization of generation ramping rates beyond traditional elastic limits [3]. To meet the changes in the system net load, the system operator can redispatch fast response units like gas turbines while maximizing the use of cheap base load units like nuclear and coal. An example of such an economic dispatch model while considering ramping rates in the fuel cost function is given in [4].

While adding renewable generation to the resource pool will significantly affect the total generation cost, as has been reported in previous works [5], it also offers opportunities for utilities to lower their carbon footprint. This directly translates into monetary savings that could be achieved in the presence of policies that control carbon pricing such as carbon tax. However, the frequent ramping up or down of the dispatchable thermal power plants to meet the system net load may also lead to increased emissions, thus exposing electric utility to additional losses due to carbon emissions. Some early work that investigates the impact of gas turbine ramping on the carbon emissions is presented in [6], [7]. These studies utilize wind or solar photovoltaic at one-minute and fiveminute resolutions respectively and heat data from natural gas generators to assess the impact on the emission reduction. Both studies have found evidence for overall reduction of carbon emissions due to the addition of solar generation. The previous work on this topic does not consider the marginal cost of carbon and the changes in the total generation cost with different 
penetration levels of utility-scale renewable energy deployment.

The decision whether or not to invest in renewable energy to offset the cost incurred due the increased emissions is a techno-economic one and should be addressed by considering various technical and economic aspects of the power system operation and planning. In this work, we aim to investigate the impact of the utility scale solar energy deployment on the total generation costs and the emissions profile, and to propose a framework for assessment of profitability of investing in intermittent renewable energy, especially utility-scale solar generation to meet carbon footprint targets.

\section{Problem Statement}

The objectives of this study are a) to study the impact of geographically dispersed utility-scale solar generation on the resource allocation and b) to investigate the changes in the carbon footprint of the existing resource pool by adding solar generation to the mix. We propose to address the economics-related part of the problem by observing the changes incurred on the marginal cost of carbon as the penetration of the solar generation in the energy mix changes. The emissions are estimated based on the generation profile of the non-renewable sources. This is accomplished by running optimal dispatch on the coal fired plants and gas turbines while considering the ramping costs in the optimization process. The optimal dispatch is run to meet the system net demand which is obtained by subtracting the net solar generation output of the geographically dispersed utility-scale plants from the actual system load. To obtain the system net demand we propose the use of Markov Chain Monte Carlo (MCMC) simulations since the output of a photovoltaic system has been consistent with Markovian dependence [12].

\section{Markov Chain Solar Modeling}

The output of a solar photovoltaic system appears to be more stable when viewed in hourly intervals. However, due to the transient cloudy conditions and random weather disturbances, the generation output of a typical photovoltaic system usually suffers rapid variations. To estimate the impact of adding solar energy to the existing generation pool, it is thus crucial that the intermittency of the incident radiation, when viewed in sub-hourly time scales, be appropriately accounted for. Since the availability of high-resolution solar data that adequately captures the sub-hourly variations in the solar insolation incident on the solar panel, it becomes imperative to use stochastic techniques to synthesize data with a very high temporal resolution. In this study we utilize the hourly averaged Typical Meteorological Data (TMY3) [8] to generate the high resolution minute-by-minute solar irradiance profile. The Markov weather model used to generate changes in the solar insolation with a temporal resolution of one minute from the given averaged hourly values is described in Figure 1. The model is initialized by calculating the average hourly clearness index $k_{t}$. The clearness index $k_{t}$ is defined as the ratio of measured irradiance $E_{m}$ at earth's surface and the irradiance that corresponds to cloudless conditions at the same location. This is referred to as clear sky irradiance $E_{\text {clear }}$ :

$$
k_{t}=\frac{E_{m}}{E_{\text {clear }}}
$$

The accurate estimation of the clear sky irradiance $E_{\text {clear }}$ is very important and has a significant influence on the clearness index. The clear sky irradiance is estimated based on the procedure given in [9]. The model works by using the hourly $k_{t}$ values as input and the sub-hourly transitions of the clearness index are determined by utilizing the transition probabilities which are extracted from the clearness index, although at a higher temporal resolution as opposed to the hourly $k_{t}$ values. This information is passed on to a first order Markov process which estimates the next state of the clearness index (sub-hourly) based on the current state (hourly). Assuming that the clearness index can assume a total of $n$ states, the transition probability of the first order Markov process can be described by the following equation

$$
P\left(k_{t}(n+1)=j \mid k_{t}(n)=i\right)=p_{i j}
$$

These transition probabilities are determined based on changes in the $k_{t}$ values that are generated at a higher temporal resolution which is the same as the desired resolution of the model output. The transition probabilities thus calculated are grouped together in a transition probability matrix, an $n$ by $n$ matrix with $n$ representing the total number of states, of the form

$$
P=\left[\begin{array}{ccc}
p_{11} & \cdots & p_{1 n} \\
\vdots & \ddots & \vdots \\
p_{n 1} & \cdots & p_{n n}
\end{array}\right]
$$

The transition probability matrix in (3) is a stochastic matrix since the cumulative probabilities of each row sum to one.

$$
\sum_{j=1}^{n} p_{i j}=1 ; i=1,2 \ldots, n
$$




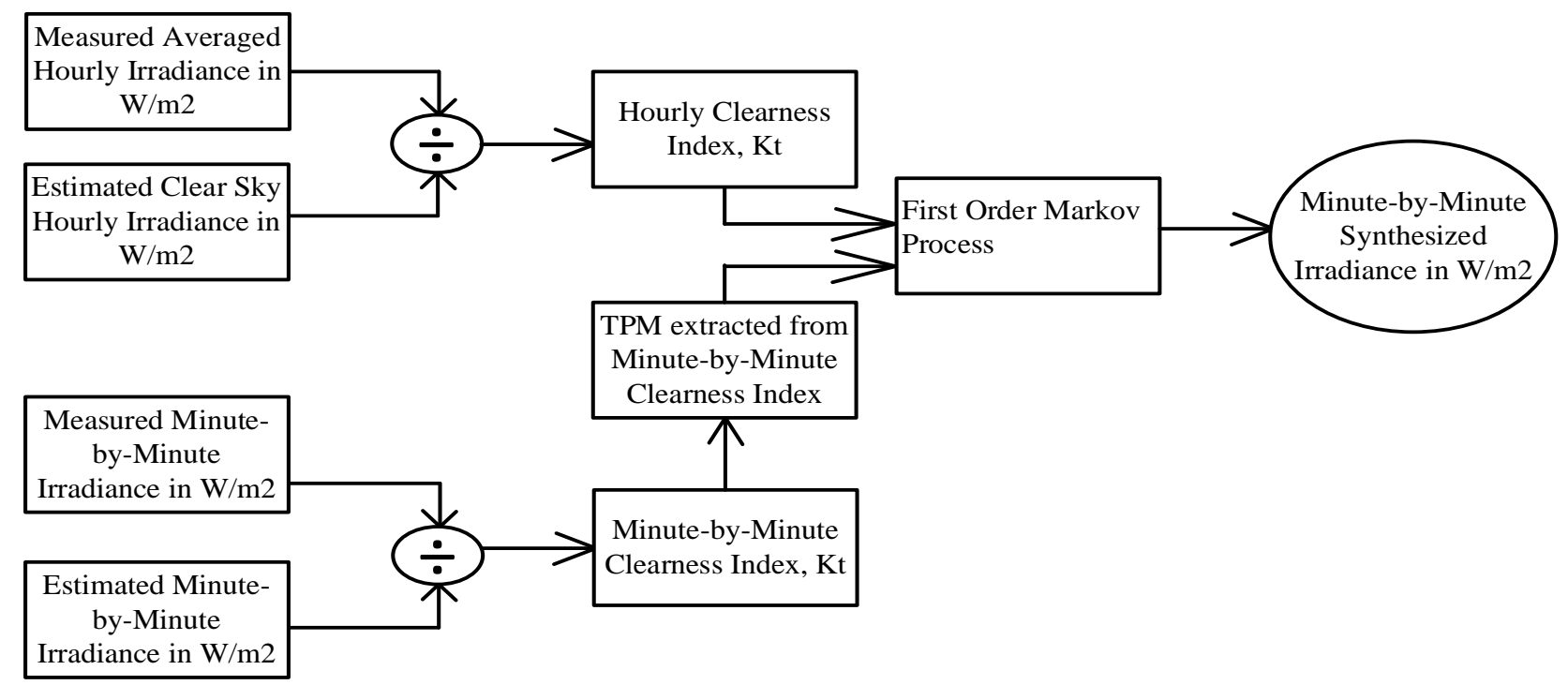

Figure 1 Markov Weather Model

The final step of the model is executed by running a Monte Carlo simulation of the Markov process. This is accomplished by sampling a uniform random number $u \sim$ Unif $(0,1)$ in the open interval $(0,1)$ and comparing with the cumulative probability of each row in the transition probability matrix. For instance, if the current state of the clearness index is $i$, the cumulative probability of state $i$ can be determined by summing the transition probabilities of the $i^{t h}$ row in the transition probability matrix. If $j$ and $m$ are two consecutive states and $k_{t}(n)=i$, the clearness index at the next time step $k_{t}(n+1)=m$ if

$$
F_{i j}<u \leq F_{i m}
$$

In (5), $F_{i m}$ is the cumulative probability of state $i$ and is given by

$$
F_{i m}=\sum_{k=1}^{m} p_{i k}
$$

An example of the transition probability matrix extracted from the sub-hourly clearness index values is shown in Figure 2. The sub-hourly clearness index values are calculated based on the measured global irradiance and the estimated clear sky irradiance of the Milford area in Utah. The raw data used to extract the transition probabilities has a temporal resolution of one minute and was retrieved from the NREL solar database [8]. The diagonal dominance of the transition probability matrix is evident with some outliers that indicate rapid changes in the clearness index. The vertical axis represents the current state of the clearness index and the horizontal axis is the next state. The clearness index takes values in the interval $[0,1]$. The colored boxes in Figure 2 represent the corresponding transition probabilities which are color

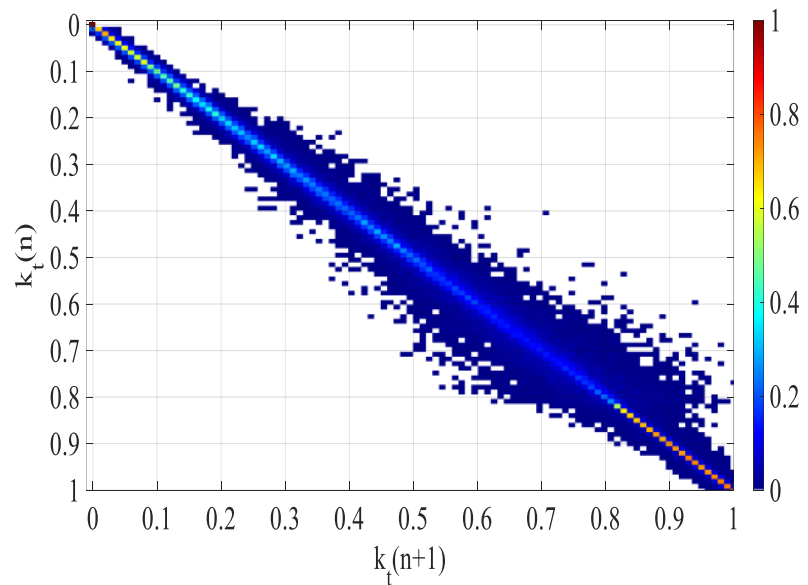

Figure 2 Representation of TPM indicating changes in $\mathbf{k}_{\mathbf{t}}$

coded and can read off the color bar. From Figure 2 it can be inferred that the highest transition probabilities correspond to clear sky index to stay the same or change very slightly. The same is true for a cloudy day with $k_{t}=0$ thus giving the transition matrix a diagonal structure overall where the largest probabilities occur on the diagonal.

The transition probability matrices thus obtained are used as an input to initiate the Markov Chain Monte Carlo simulations. The MCMC simulation algorithm combines the transition probabilities generated at sub-hourly time scales with the hourly average TMY3 meteorological weather data to generate high resolution irradiance profiles. We use the hourly averaged TMY3 weather data of the seven representative sites in the south east region of the United States. The PV systems at each of the representative sites are oriented at an azimuth of $180^{\circ}$ 
(south-facing) with a panel tilt of $30^{\circ}$. The capacity of the each solar farm is chosen to be proportional to the population of each site in 2019.
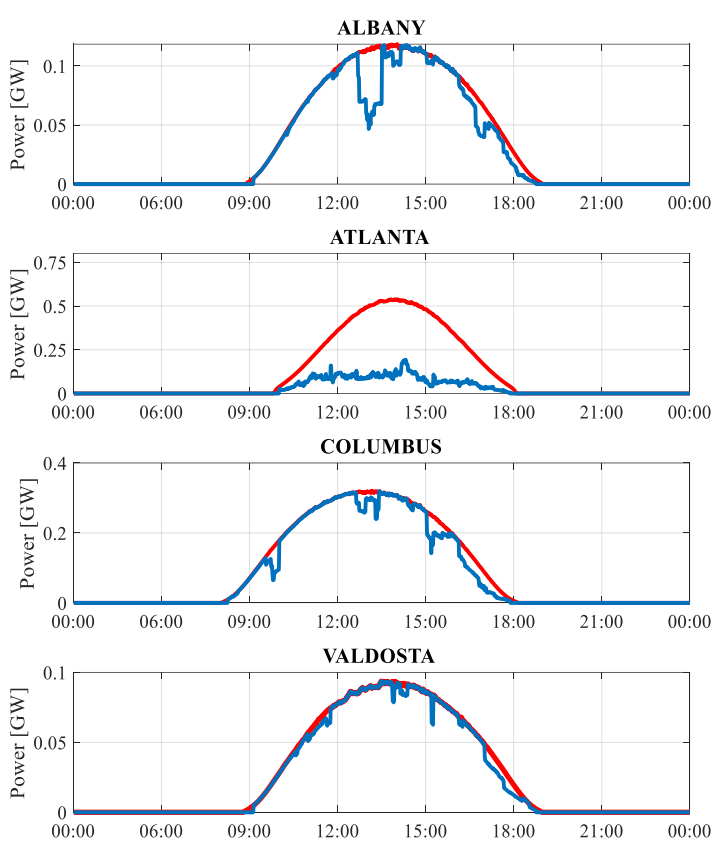

patterns which in turn depends on the spatial spread of the cluster. For the seven representative sites shown in Figure 3 the aggregated output on the same day is
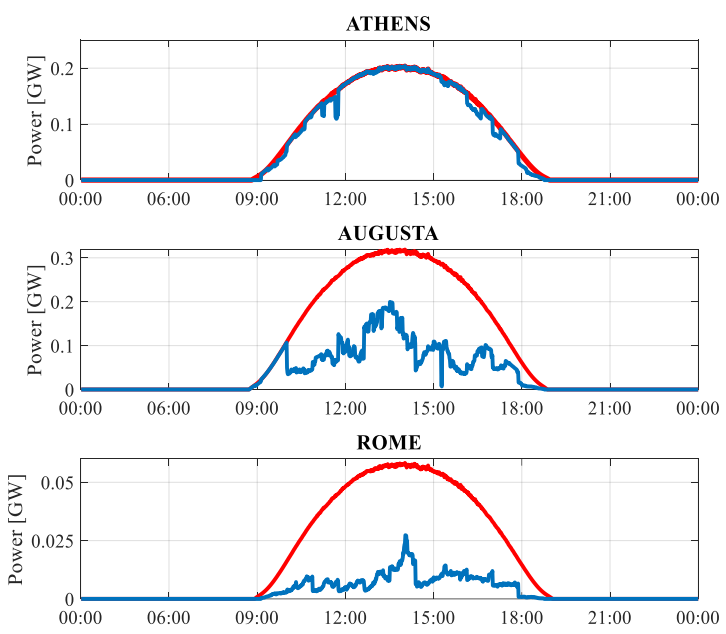

\section{Figure 3 Generation output of seven sites in the SE region. Red curves represent clear sky conditions and the blue curves show the impact of transient clouds.}

The solar output of each representative site show significant variability and it is expected when the generation outputs are averaged over a number of solar farms that are dispersed geographically, the variability in the production will decrease. The reduced variability in the aggregated output however will depend on the measure of similarity in the production

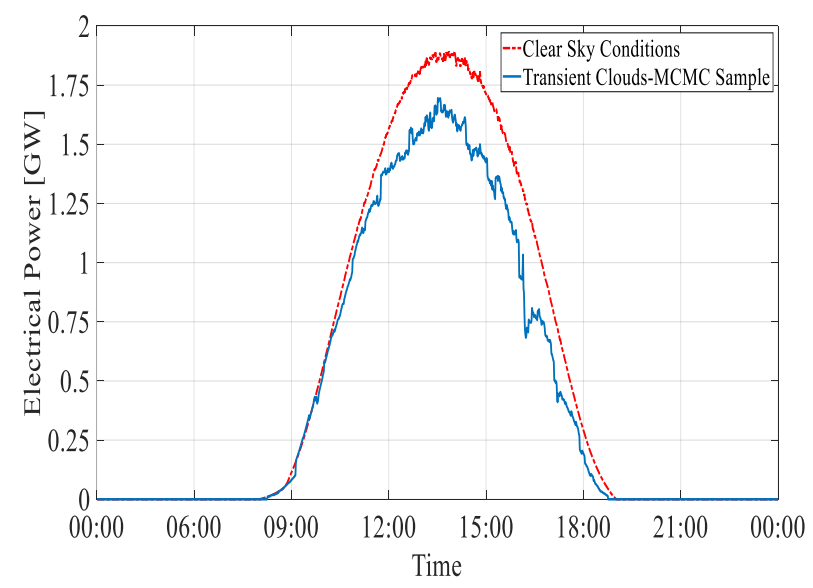

Figure 4 Combined output of seven representative sites in SE region shown in Figure 4. The red curve represents the aggregated output for clear sky conditions while the blue curve is the aggregated generation output that accounts for the cloud movement. Figure 4 shows a significantly smaller variation in the generation output as compared to any of individual solar farms in Figure 3 on a transient cloudy day.

\section{Framework for Optimal Dispatch}

The synthesis of the solar irradiance with high temporal resolution is essential for estimating the output of the solar generation plant. The solar output in turn is used to estimate the system net load. The dispatchable resources, such as coal and gas plants are then considered in the optimization of generation allocation to meet the system net load. In this work, we use the fuel cost model presented in [4]. This model accounts for the ramping of fast dispatchable units like gas turbines by adding a ramping cost term to the conventional quadratic cost function. The cost functions for coal and gas generators used in the optimization process are

$$
C_{i, \text { coal }}=\alpha+\beta P_{g i}+\gamma P_{g i}^{2}
$$




$$
C_{j, g a s}=\alpha+\beta P_{g j}+\gamma P_{g j}+d \frac{d P_{g j}}{d t}
$$

Since coal fired plants do not possess a fast-ramping capability, the ramp rate constraint is not considered for the coal generators. The gas generators on the other hand due to their fast-ramping capability are allowed to follow the load. The coefficients in the fuel cost model are computed by using the method of least squares applied to the real data. The coefficient of the ramp rate constraint $d$ is identified separately depending on whether the output is decreasing or increasing. Table I lists the values of the cost coefficients as reported in [10].

Due to the variable output of the solar generation, the system net load experiences fast changes which need to be compensated to satisfy the system demand at each time instant. While this compensation is achieved by regulating the output of the gas turbines, the formulation of the cost function levies a heavy penalty for an increasing ramp rate when compared with a decreasing ramp rate.

Table I Coefficients of Fuel Cost Model [10]

\begin{tabular}{|c|l|l|}
\hline Coefficient & \multicolumn{1}{|c|}{ Coal } & \multicolumn{1}{c|}{ Gas } \\
\hline$\alpha$ & 3.1626 & 11.908 \\
\hline$\beta$ & 0.19499 & 0.0684 \\
\hline$\gamma$ & 0.000023 & 0.000082 \\
\hline$d$ & - & $0.03 ; \frac{d P_{g i}}{d t}>0$ \\
& & $0.018 ; \frac{d P_{g i}}{d t}<0$ \\
\hline
\end{tabular}

The base load data with zero percent photovoltaic is obtained from EIA grid monitor website [11]. To better appreciate the energy consumption of the southeast region in 2019, the load duration curve (LDC) is shown in Figure 5. The base LDC curve is derived from the actual net generation and demand of the southeast region which in the year 2019 stood at $252,490 \mathrm{GWh}$ and $243,140 \mathrm{GWh}$ respectively and peak load of approximately $46 \mathrm{GW}$. Figure 5 also plots the LDC curves corresponding to different penetration level of the utility scale solar generation. The addition of the increasing solar generation to the existing resource portfolio decreases the system effective load as in Figure 5.

The economic dispatch problem to meet the system net load for different penetrations of solar generation is formulated as

$$
\begin{gathered}
\text { minimize } \sum_{k=1}^{\mathrm{K}} \sum_{i=1}^{N} C_{i}\left(P_{g i}\right)+\sum_{k=1}^{\mathrm{K}} \sum_{j=1}^{M} C_{j}\left(P_{g j}\right) \\
\text { subject to } \sum_{\substack{i=1 \\
N}} P_{g i}+\sum_{j=1}^{M} P_{g j}-P_{D}=0 \\
P_{g i}^{\text {min }} \leq P_{g i} \leq P_{g i}^{\text {max }} \\
P_{g j}^{\text {min }} \leq P_{g j} \leq P_{g j}^{\text {max }}
\end{gathered}
$$

In (9), $P_{D}$ refers to the system net load and approximate losses, $N$ is the total number of coal

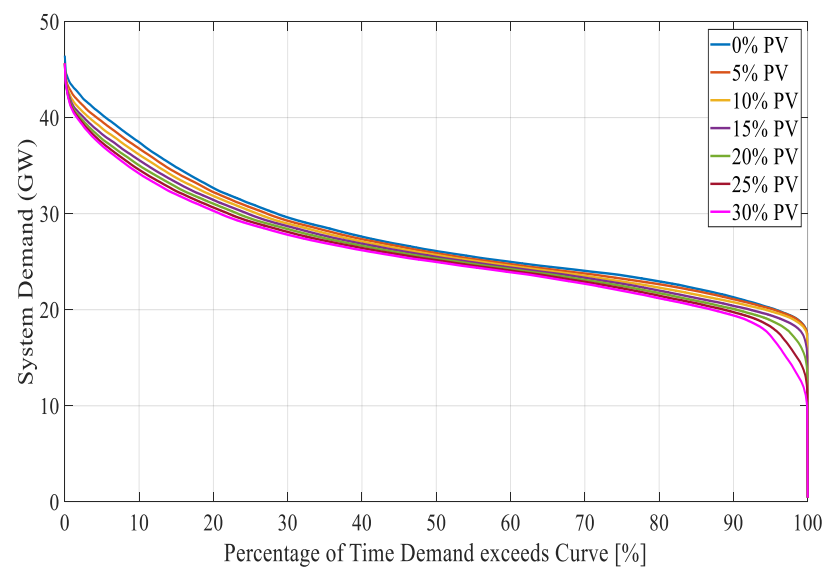

Figure 5 Load Duration Curves for base load and with PV

generators, $P_{g i}$ is the output of $i^{\text {th }}$ coal generator, $M$ is the number of gas generators, $P_{g j}$ is output of the $j^{\text {th }}$ gas generator and $k$ is the optimization period. Since losses are non-essential to this study we choose to consider them constant. This simplification does not necessarily affect the conclusions of this study, but should be addressed in practice as it is impacted by locations of plants and loads as well as by network topology. We define the augmented cost function in (10). The optimal solution of the constrained problem can be found from the Kuhn Tucker conditions. The search for the optimal solution continues until an acceptable tolerance is met.

$$
\begin{aligned}
\widetilde{C_{T}}=\sum_{k=1}^{\mathrm{K}} & {\left[\sum_{i=1}^{N} C_{i}\left(P_{g i}\right)+\sum_{j=1}^{M} C_{j}\left(P_{g j}\right)\right]-} \\
\lambda & {\left[\sum_{i=1}^{N} P_{g i}+\sum_{j=1}^{M} P_{g j}-P_{D}\right] }
\end{aligned}
$$




\section{Case Study}

To assess the impact of utility-scale solar deployment on the generation costs and emissions the study assumes that solar plants are installed across the southeast region of the U.S. The study uses the energy mix of the southeast region as an input for running the economic dispatch. Table II lists the energy mix of the SE region as reported by EIA for the year 2019. The data used pertains to the fuel type, percentage of the mix and operating costs of generation specific to fuel type in $\$ / M W h$. The study uses the unsubsidized average levelized cost of energy of the solar generation [1].

Table II Energy Mix of SE Region, 2019 [11]

\begin{tabular}{|l|l|l|l|l|}
\hline \multicolumn{1}{|c|}{ Type } & $\begin{array}{c}\text { Gen } \\
(\mathrm{TWh})\end{array}$ & Fuel & $\%$ Mix & \$/MWh \\
\hline Base & 47.719 & Nuclear & 18.89 & 10.63 \\
\hline $\begin{array}{l}\text { Inter- } \\
\text { mediate }\end{array}$ & 57.225 & Coal & 22.64 & 21.17 \\
\hline & 12.982 & Hydro & 5.14 & 6.86 \\
\hline Peak & 129.54 & Gas & 51.03 & 22.57 \\
\hline & & PV & - & $31-42$ \\
\hline
\end{tabular}

The sites chosen for the installation of the solar plants are given in [12]. It is assumed that all solar plants are geographically dispersed across the SE region. The solar generation is simulated in capacities ranging from $5 \%$ to $30 \%$ of the annual peak demand. This translates to a total solar capacity of $2.25 \mathrm{GW}$ corresponding to $5 \%$ penetration and $13.5 \mathrm{GW}$ for $30 \%$ penetration. The individual PV modules are oriented at an azimuth of 180 degrees (south facing) and tilt angle of 30 degrees. To simulate the solar generation the study collects the hourly TMY3 solar insolation data of the representative sites and estimates the hourly clearness index values. The National Solar Radiation Database [8] contain high resolution data for the state of Georgia and Alabama. Hence the study uses the high resolution data from seven test sites from 2010-2012 [12]. The transition probability matrix from the Milford area in Utah is chosen to synthesize the high resolution clearness index for the representative sites across the SE region since the annual output of Utah most closely resembles that of Georgia. The solar output in minute intervals is estimated using PV_LIB [13]. The study assumes Canadian Solar CS5P-220M solar modules and Siemens SINVERT PVS 1401 UL inverters.

The study utilizes annual simulations of the net generation as well as generation by source and net system demand of the SE region for the year 2019.

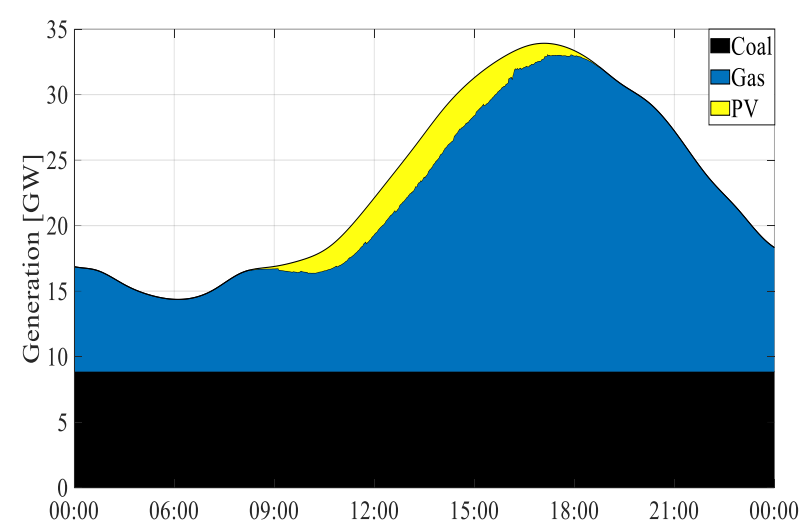

Figure 6 Generation Profile with 10\% UtilityScale Solar

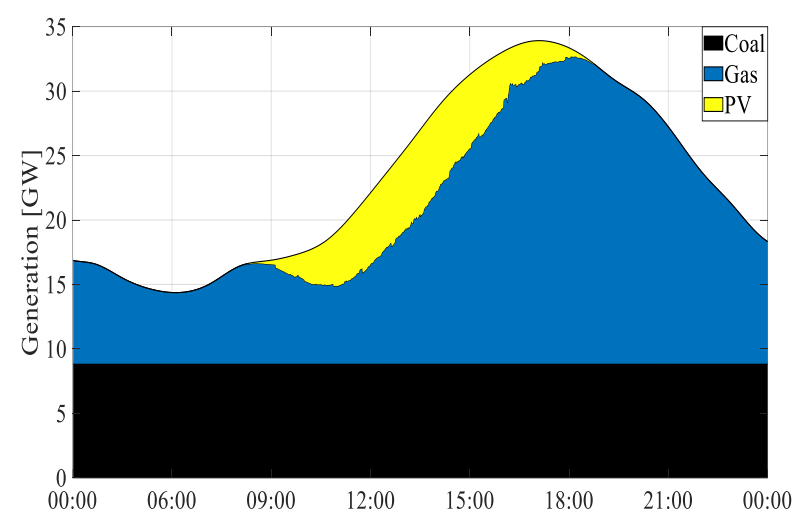

Figure 7 Generation Profile with $20 \%$ UtilityScale Solar

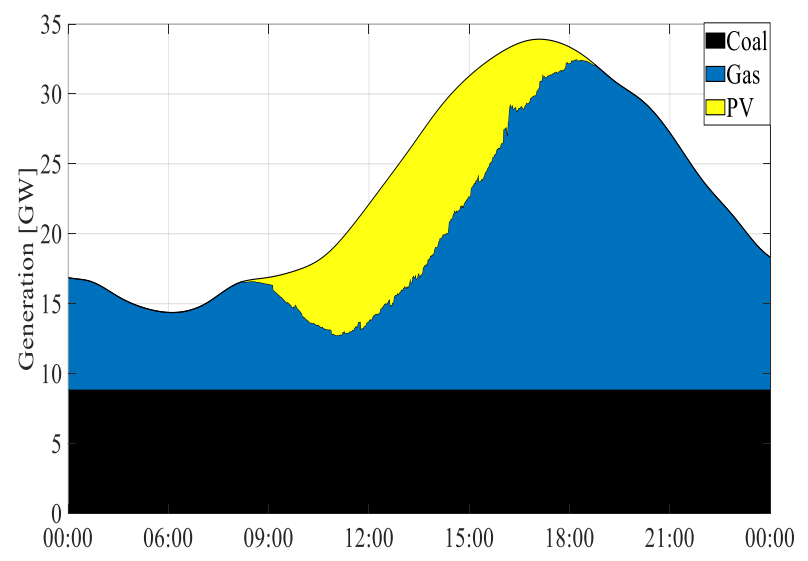

Figure 8 Generation Profile with $30 \%$ UtilityScale Solar

The results of the generation allocation when the flexible sources like gas generation are dispatched to meet the net load are shown in Figure 6, Figure 7 and Figure 8 . The generation profiles shown represent the day with some transient cloud activity that corresponds to the aggregate generation output shown 
in Figure 4. Since nuclear is the base unit with little or no impact on the emissions results of resource allocation are shown only for coal, gas and solar generation. When the generation pool is assumed to consist of solar energy the output of the gas turbine exhibits ramping characteristics and the magnitude of the ramping increases with the increase in the penetration of solar generation. The results shown in Figures 6, 7, 8 do not consider ramping constraints on coal since load following by coal is very expensive and large units may undergo significant damage due to fast ramping. The gas turbine is used to follow the load and is assumed to have fast ramping capability. The gas output increases when the solar production drops to make up for the deficit. Similarly, when the solar generation increases the gas output ramps down to balance the load. The frequent ramping up and down of the more expensive gas along with the cost of solar generation has the impact of increasing the total cost of generation. As the share of solar continues to grow, at one point solar will start pushing nuclear out. At that point, the system will need more coal and gas to balance solar and the total $\mathrm{CO}_{2}$ emissions would start rising. Such "renewable paradox" (i.e. increasing emissions as more RE is added to the system) is already noticed in Ukraine where nuclear accounts for about $50 \%$ of power generation.

The annual cost of generation of coal, gas and PV when no constraints are applied to the coal output is shown in Figure 9. The cost of generation increases with penetration level since utility-scale photovoltaic is still the most expensive source of generation as compared to coal and gas. However, since the ramping constraints on coal are ignored and given that coal is least expensive, the presence of additional solar generation leads to an overall decrease in the more expensive gas generation in order to balance the load.

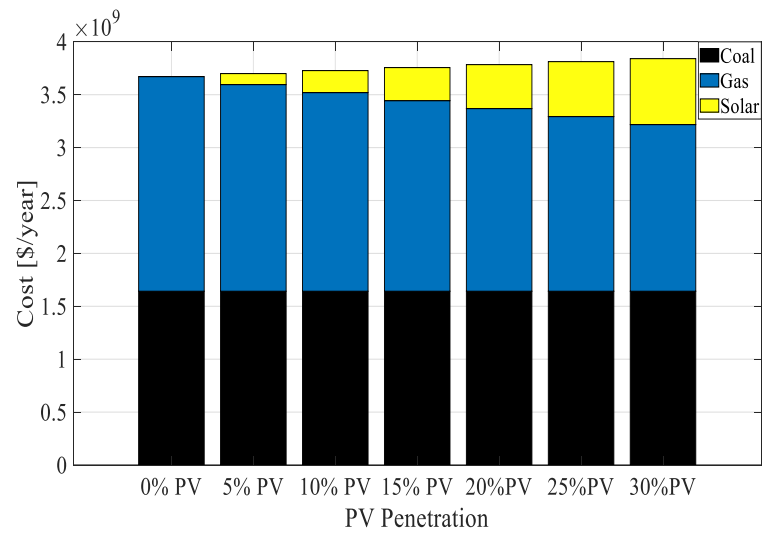

Figure 9 Annual Generation Cost without Coal Retirement
Overall, the cost of generation increases with the addition of solar to the mix.

Figure 10 shows the annual generation costs when the output of coal is constrained and the amount of coal retired is set equal to the peak solar generation at each penetration level[14]. The generation costs in this case are observed to increase at a faster rate than in the case in which no ramping constraints were applied to the coal generation. Since coal is progressively retired the amount of gas generation required to support the solar increases at each penetration level. The overall generation costs do not exhibit an exorbitant rise as has been reported earlier. This can be attributed to the significant decline in the generation costs of the utility-scale solar plants. As a result, the relevance of the solar generation costs to act as a deterrent to the wide-spread adoption of solar generation is rapidly wearing down. What is concerning, however, is the economic impact of frequent ramping up and/or down of the gas turbines to compensate for the variability of the solar generation.

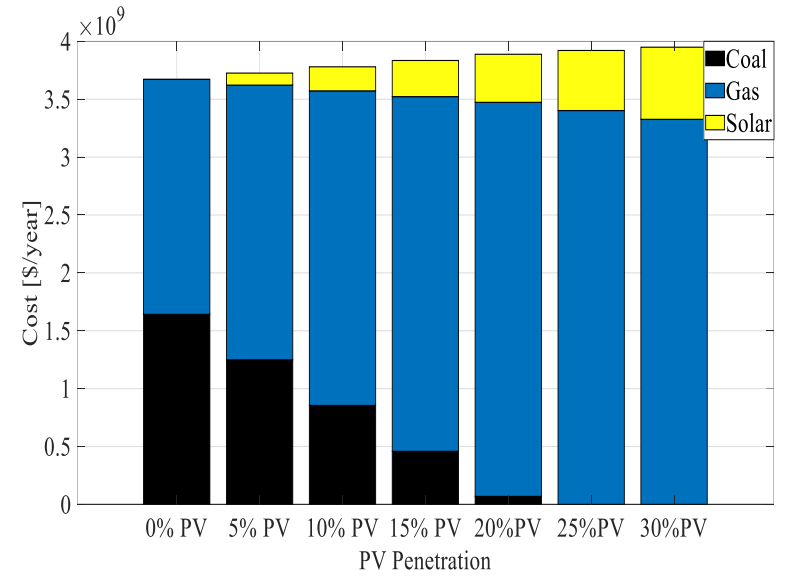

Figure 10 Annual Generation Costs with Coal Retirement

To make up for the rapid changes in the solar output the gas spinning reserve is set equal to the maximum change in the solar generation for a given day. The spinning reserve is thus scheduled at every minute to offset the changes in the solar output. This however results in increased emissions and increased ramping costs of the gas turbine. The relative ramping costs of gas at different penetration levels of solar can be visualized in the form of the bar graph in Figure 11. Although the cost of gas ramping is small as compared to the total operating cost of fuel, it increases with additional solar deployment. This is expected since higher capacity solar will result in higher changes in the system load. 


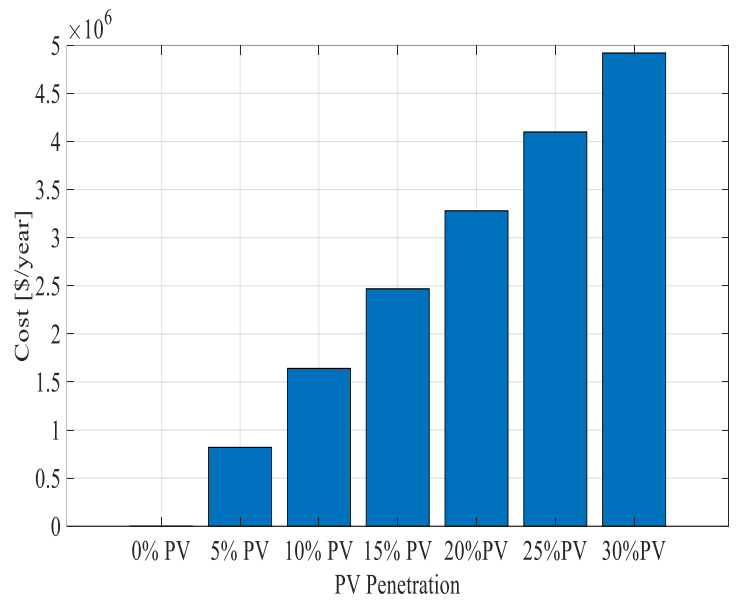

Figure 11 Annual Ramping Costs of Gas Turbines

$$
S R=\max \left\{\frac{\Delta P_{g}^{P V}}{\Delta t}\right\}
$$

From the emissions perspective, the deployment of additional solar capacity reduces the amount of pollutants and greenhouse gases by reducing the dependency on fossil-fuels. To estimate the amount of carbon emitted in the form of carbon dioxide in metric tons this study used the emission data as reported by EIA [15]. The amount of emission is estimated based on the emission coefficients of each fuel type. Figures 12 and 13 show the amount of carbon emitted in metric tons for one day with and without coal retirement. When the ramping constraint on coal is ignored the carbon footprint of the gas generation decreases with the increase in peak solar capacity (Figure 12). On the

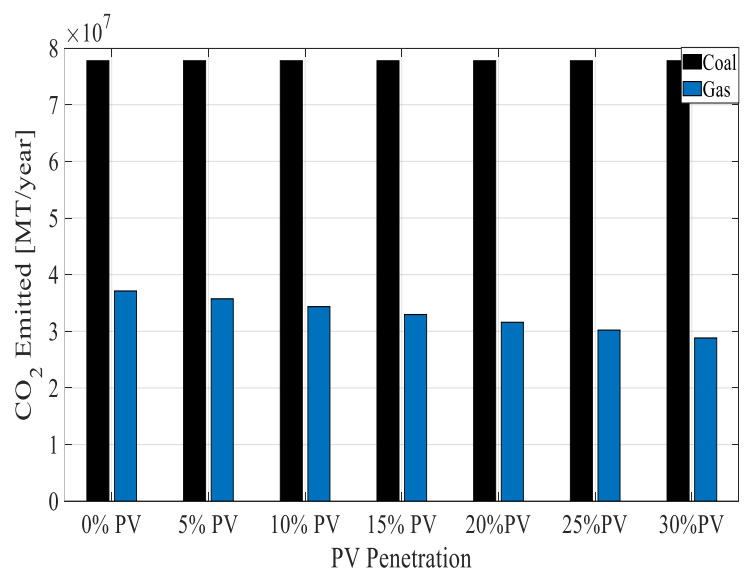

\section{Figure 12 Carbon Emitted in MT/year without Coal Retirement}

other hand, when coal is retired, as additional solar capacity is added to the system, the generation and carbon emissions of gas-fired plants increase with the increasing solar capacity (Figure 13).

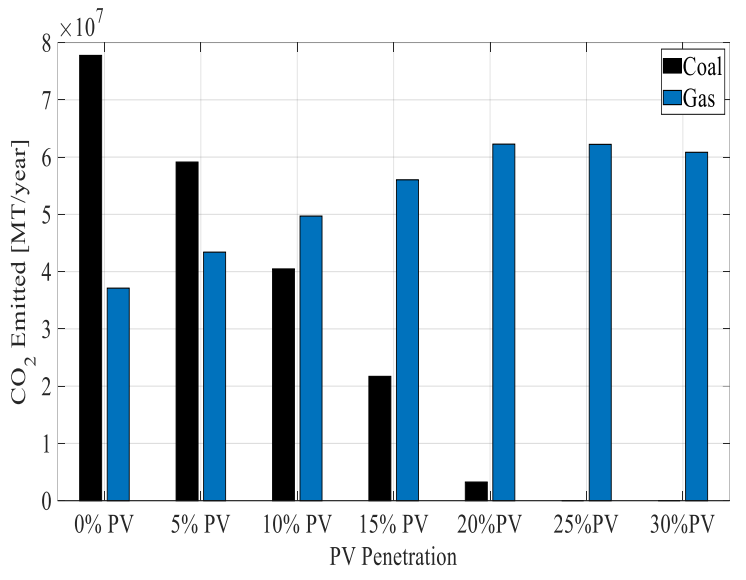

\section{Figure 13 Carbon Emitted in MT/year with Coal Retirement}

To estimate the costs related with the carbon emissions and also to investigate the impact of the carbon tax policies we estimate the emission costs for a range of carbon tax values in interval [ 1100$]$ 10/MT. The resulting range of emission costs as a function of solar penetration are shown in Figure 14 and Figure 15. The minimum value in the box plots of Figure 14 and Figure 15 for each penetration level corresponds to the carbon tax of $\$ 1 / \mathrm{MT}$ and the maximum value corresponds to the carbon tax of $\$ 100 / \mathrm{MT}$. When the output of coal fired plants is held constant as the penetration of solar is progressively increased, the range of cost emissions narrows as the solar penetration increases. However, the constriction of the range of cost emissions is much more pronounced when the coal is progressively retired as opposed to not retiring coat at all. This is mainly because the emission coefficient of coal-fired generation is 2.21 pounds per $\mathrm{kWh}$ from coal which compares to 0.91 pounds per $\mathrm{kWh}$ from gas-fired power generation [1].

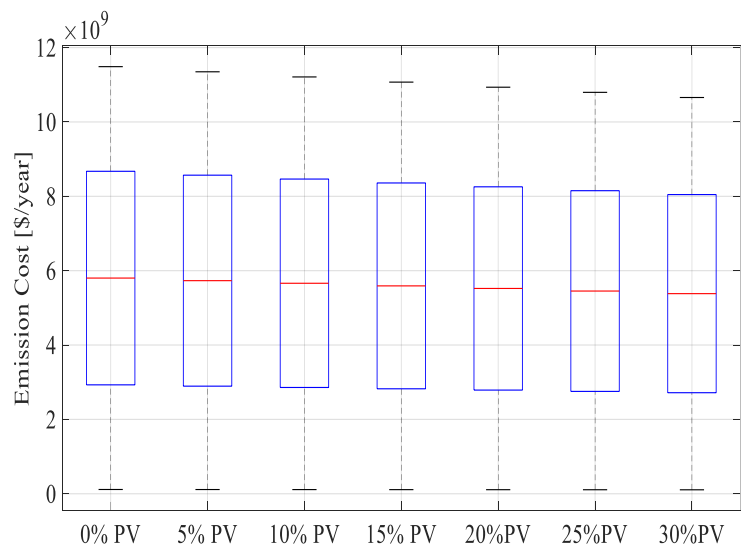

Figure 14 Range of Cost Emissions (\$/yr) without Coal Retirement 
With subsequent retirement of coal (Figure 15) the cost of carbon emissions incurred to the utility decreases by more than $50 \%$ as the solar penetration level increases from $0 \%$ to $30 \%$ of the annual peak demand.

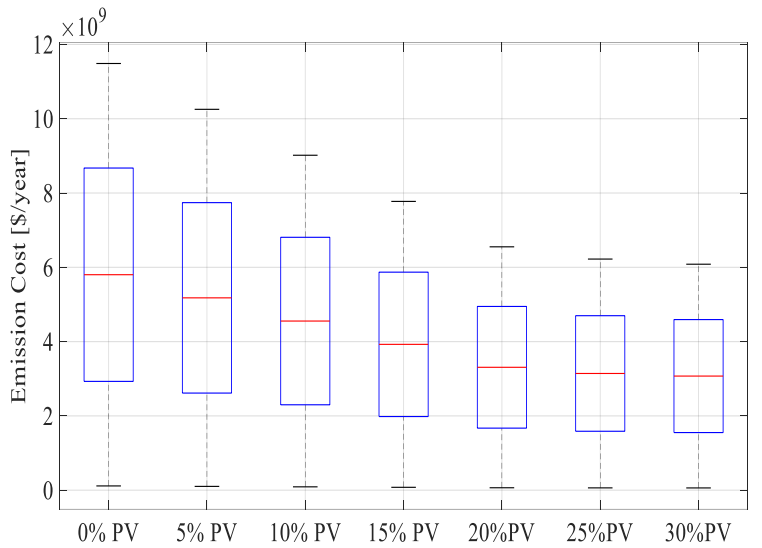

Figure 15 Range of Cost Emissions (\$/yr) with Coal Retirement

An interesting aspect of this study is the investigation of the impact that frequent ramping of gas turbines can have on the benefit in terms of carbon emissions that is expected from the deployment of solar generation. To address this the study calculates the annualized marginal benefit of carbon abatement which is defined as the ratio of change in the annual cost of carbon emissions and corresponding change in the annual generation costs as a function of carbon tax rates and solar penetration. The addition of renewable generation to the existing generation pool is predicated on the assumption of lowering the carbon emissions and hence the costs associated with the emissions. However, since solar generation is more expensive than coal and/or gas, the addition of solar to the mix increases the overall generation cost. Moreover, at higher penetrations of solar generation, the frequent ramping up and down of gas turbines further diminishes the savings in terms of cost emissions. The marginal benefit curve when plotted as a function of carbon tax rates and solar penetration can be used to investigate the profitability of adding solar generation to the mix. The marginal benefit as a function of carbon tax rate and solar generation without and with coal retirement is shown in Figure 16 and Figure 17 respectively.

From Figure 16 and Figure 17 we conclude that for a fixed carbon tax rate the marginal benefit decreases with increase in solar generation. Moreover, the decrease is more rapid at higher carbon tax rates than at lower carbon tax rates. This is because increasing the solar generation also increases the frequency and the magnitude of the gas turbine ramping which results in increased carbon emissions thereby rapidly curtailing the benefits of adding solar to the mix. Furthermore, when viewed as a function of carbon tax alone, the marginal benefit decreases with the increase in carbon tax rate up to a certain point and then begins

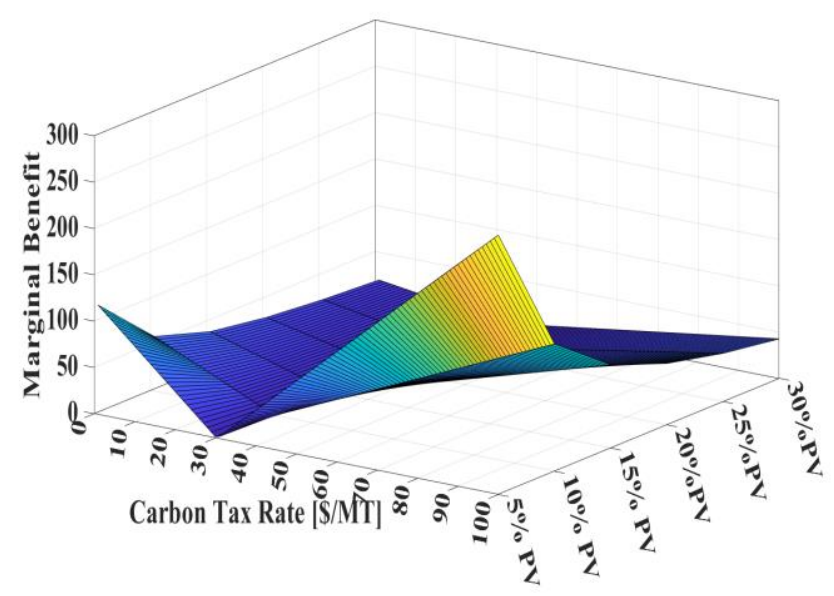

Figure 16 Marginal Benefit of Carbon abatement without Coal Retirement when seen as a function of Carbon Tax Rate and PV Penetration

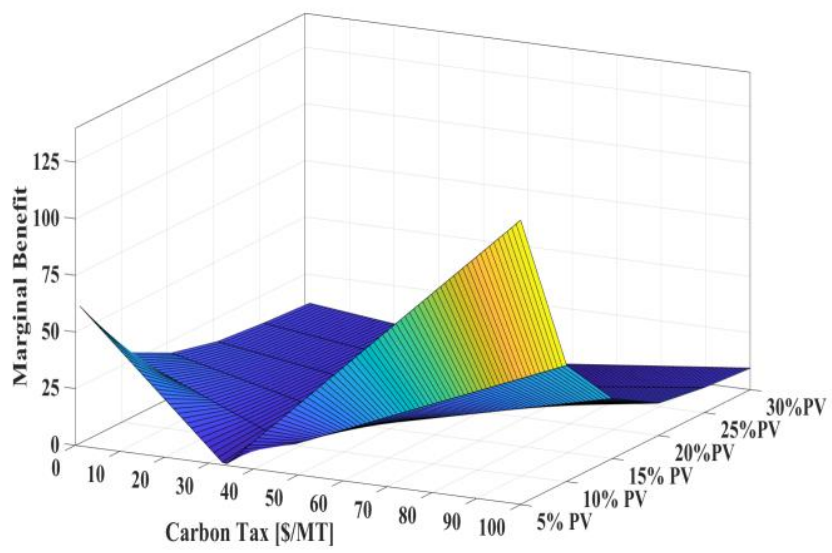

Figure 17 Marginal Benefit of Carbon abatement with Coal Retirement when seen as a function of Carbon Tax Rate and PV Penetration

to increase as the carbon tax is further increased. When coal retirement is not considered this inflection point where the curvature changes sign corresponds to a carbon tax rate of $\$ 31 / \mathrm{MT}$ which coincides with the levelized cost of solar generation. Also, the inflection point is the same for all levels of installed solar capacity in the scenario where coal generation is not retired. However, when coal retirement is considered as shown in Figure 17, the inflection point corresponds to different carbon tax rates for different solar capacity levels; progressively increasing as the solar capacity increases. This seems to suggest that a fixed carbon tax will result in a decreasing or at best a constant 
marginal benefit with increasing penetration of solar generation. On the other hand, a variable carbon tax rate, one that changes with the change in the installed solar capacity can result in a marginal benefit that increases as the installed solar capacity increases.

\section{Conclusions}

The purpose of the study is to investigate various technical and economic challenges associated with a widespread adoption of intermittent renewable energy. The increasing intermittent generation has a significant impact on the allocation as well as the carbon footprint of conventional resources. For example, it is shown that a higher penetration of solar generation significantly increases the cycling of the gas turbines and the magnitude of cycling is more pronounced with the subsequent retirement of coal fired plants. The frequent cycling of the gas turbines could lead to an increased wear and tear of the equipment especially the high temperature components in a phenomenon referred to as creepfatigue interaction [4].

From the emissions and economics standpoint the addition of solar generation results in an overall net reduction in the emission costs but increases the amount of gas ramping required to balance the variable net load. This further increases the ramping costs and carbon emission thereby resulting in an exponential decline of marginal benefit of carbon abatement derived by adding solar generation to the existing generation portfolio.

The results presented call for a more nuanced and a multifaceted approach towards the management of intermittent generation, load following and the scheduling of spinning reserve. From a utility perspective the end goal is to operate the system at the optimal economic point that would not impact the system reliability while at the same time keeping the overall costs low enough not to exceed the cost of alternative solutions. From the point of view of policy makers and regulators, it is important to recognize that targets set for specific technology (e.g. use of solar PV) to decarbonize the power sector cannot be applied as a "one size fit all" across different power utilities. Each power utility, depending on the energy mix of its power generation, faces specific task of finding an optimal level of penetration of renewable energy sources for the given cost of carbon. As the cost of carbon (e.g. carbon price or tax) increases, some utilities will be able to integrate more renewables in a cost-effective manner faster than others. This calls for a careful planning and coordination between policy makers, regulators, and utilities to ensure that consumers pay the least cost for energy transition towards a carbon neutral power sector.

\section{References}

[1] "Energy Information Agency: Today in Energy," EIA, 2021. [Online]. Available: https://www.eia.gov/todayinenergy/detail.php?id= 46416.

[2] M. Liu, P. Reed, and C. L. Anderson, "Stochastic Synthetic Data Generation for Electric Net Load and Its Application," in Proceedings of the 54th Hawaii International Conference on System Sciences, 2021.

[3] G. B. Shrestha, K. Song, and L. Goel, "Strategic self-dispatch considering ramping costs in deregulated power markets," IEEE Trans. Power Syst., 2004.

[4] R. Bhat, M. Begovic, I. Kim, and J. Crittenden, "Effects of PV on conventional generation," in Proceedings of the Annual Hawaii International Conference on System Sciences, 2014.

[5] I. Kim, M. Begovic, H. Jeong, and J. Crittenden, "Impact of photovoltaic distributed generation on generation resource allocation," in Proceedings of the Annual Hawaii International Conference on System Sciences, 2013.

[6] W. Katzenstein and J. Apt, "Air emissions due to wind and solar power," Environ. Sci. Technol., 2009.

[7] S. Blumsack and K. Richardson, "Cost and Emissions Implications of Coupling Wind and Solar Power," Smart Grid Renew. Energy, 2012.

[8] S. Wilcox, "National Solar Radiation Database 1991-2010 Update: User's Manual ," Nrel/Tp5500-54824, 2012.

[9] D. A. McCracken, "Synthetic High Resolution Solar Data," 2011.

[10] T. Shiokawa, Y;Kumano, "A new fuel cost model of thermal unit considering output ramp rate and its application to Economic Load Dispatch," in " Electrical Power \& Energy Conference (EPEC), 2009, p. vol., no.pp.1,6, 22-23.

[11] "EIA Hourly Grid Monitor." [Online]. Available: https://www.eia.gov/electricity/gridmonitor/dashb oard/electric_overview/US48/US48.

[12] M. M. Begovic, I. Kim, B. Vidakovic, P. Djuric, and V. Jeremic, "Impact of Short-Term Variations in the Generation Output of Geographically Dispersed PV Systems," in Proceedings of the 50th Hawaii International Conference on System Sciences (2017), 2017.

[13] Sandia National Laboratories, "PVPMC, PV_LIB Toolbox.".

[14] D. Lew, G. Brinkman, N. Kumar, P. Besuner, D. Agan, and S. Lefton, "Impacts of Wind and Solar on Fossil-Fueled Generators," Proc. IEEE PES Gen. Meet. 2012, 2012.

[15] I. Kim and M. Begovic, "On impact of randomly distributed PV systems on distribution networks," in Proceedings of the Annual Hawaii International Conference on System Sciences, 2016. 